\title{
TESTOSTERONA, FUNCIÓN ENDOTELIAL, SALUD CARDIOVASCULAR Y ANDROGENODEFICIENCIA DEL VARON AÑOSO
}

\author{
R. Vela Navarrete, J. V. Garcia Cardoso, M. Pardo Montero, P Jiménez Máteos-Cáceres y \\ A. López Farré.
}

Servicio de Urología. Fundación Jiménez Díaz. Universidad Autónoma. Madrid.

Unidad de Investigación Cardiovascular. Hospital Clínico de San Carlos. Universidad Complutense.

Madrid. España.

\begin{abstract}
Resumen.- La determinación de testosterona en la población de varones añosos, ha demostrado su valor como marcador de salud general, no sólo sexual, motivando un mayor interés por esta determinación analítica y las potenciales interrelaciones de la testosterona con otros marcadores de salud cardiovascular, obesidad, hipertensión, disfunción eréctil, sarcopenia, síndrome metabólico, envejecimiento y otras condiciones. Se revisa de manera específica la interrelación entre salud cardiovascular, disfunción eréctil y androgenodeficien-
\end{abstract}

\section{CORRESPONDENCIA}

R. Vela Navarrete

Servicio de Urología

Fundación Jiménez Díaz

Avda. de los Reyes Católicos, 2

28040 Madrid (España)

rvelan@telefonica.net

Trabajo recibido: 24 de septiembre 2008 cia, procesos de fácil reconocimiento, prevención y tratamiento. La información actual concede tal protagonismo a la testosterona como referente de salud que su determinación parece inexcusable en la consulta del varón añoso.

Palabras clave: Testosterona. Endotelio. Androgenodeficiencia.

Summary.- Testosterone determination in an old men population has demonstrated its about the as general health marker, not only sexual, prompting a greater in to arrest for this analytic determination and the potential relations of testosterone with other markers of cardiovascular health, obesity, hypertension, erectile dysfunction, sarcopenia, metabolic syndrome, ageing, and other conditions. We specifically review the relationship between cardiovascular health, erectile dysfunction, and androgen deficiency, processes easily recognizable, prevented and treated. Current information gives such a prominence to testosterone as a health reference that its determination seems to be inexcusable in the ageing male consult.

Keywords: Testosterone. Endothelial. Androgen deficiency. 


\section{INTRODUCCIÓN}

La testosterona es el andrógeno más representativo de la masculinidad y virilidad. Estos atributos de género incluyen, entre sus expresiones fenotípicas y genotípicas, los caracteres sexuales secundarios, adquiridos progresivamente a lo largo de la vida, tales como el timbre de voz, la distribución del vello, la morfogénesis genital, masa muscular, función sexual y reproducción. Es también conocido que la testosterona no actúa de igual modo en función de la edad y de un área corporal precisa y puede hacerlo directamente, tras su conversión en DHT por la acción de la $5 \alpha$ reductasa o por aromatización estrogénica.

Muchas de las acciones de la testosterona han sido investigadas en los últimos años con gran precisión. Sin embargo, la imagen terapéutica y mediática de la testosterona, desde su descubrimiento y síntesis en 1935, ha estado ligada a sus efectos sobre la masa muscular y la resistencia física (dopaje deportivo y culturismo), así como sobre la actividad sexual, más recientemente mejor valorada al establecerse la relación entre niveles bajos de testosterona, especialmente del varón añoso, y relevantes síntomas clínicos que han constituido un síndrome, conocido con varios nombres (Andropausia, $\mathrm{LOH}$ ) y finalmente calificado como Síndrome de Deficiencia de la Testosterona (TDS) (1-3).

La investigación paralela de los niveles de testosterona y de los variados síntomas que el envejecimiento motiva en el varón añoso, ha atraído de manera extraordinaria la atención de los investigadores clínicos y en poco tiempo el TDS ha sido objeto de numerosos estudios epidemiológicos y de laboratorio, con la intención de aclarar el papel de la testosterona en los múltiples achaques del varón que envejece. De manera especial ha atraído la atención su efecto sobre la salud en general (marcador de salud y muerte prematura), sobre la salud cardiovascular y función endotelial, actividad sexual y función eréctil, además de sus conocidas acciones sobre masa muscular y fortaleza ósea.

Si un atributo característico y específico del género masculino es el vigor físico y este se consigue con buena masa muscular, es de esperar que simultáneamente exista una excelente función hemodinámica, sólo posible con buena anatomía vascular. La interrelación entre testosterona y sistema cardiovascular encuentra en esta elemental declaración de principios un fundamento de coincidencia.

En los años 40 el propianato de testosterona fue utilizado para el tratamiento de la enfermedad vascular periférica (4) y del angor pectoris (5-7) con esta hipótesis, aparentemente con buenos resultados. A principios de este siglo (8) se ha confirmado su valor como excelente vasodilatador coronario. Los pacientes con aterioesclerosis aórtica y coronaria, tienen niveles bajos de testosterona y la aterioesclerosis de la carótida progresa más rápidamente en estos individuos que en controles con niveles normales $(9,10)$. En experiencias animales la castración acelera la aterogénesis (11). Sin embargo, ha sido la investigación sobre el papel que la testosterona tiene en al función eréctil, quien ha motivado el mayor interés por esta relación entre andrógenos, disfunción endotelial y enfermedad cardiovascular.

\section{NIVELES DE TESTOSTERONA PLASMATICA $Y$ SALUD CARDIOVASCULAR}

Diversos estudios poblacionales, tanto longitudinales como transversales, han detectado una mayor frecuencia de muerte cardiovascular en los individuos con niveles bajos de testosterona plasmática (12). También lo contrario ha sido confirmado, en el sentido de que los individuos con enfermedad cardiovascular avanzada tienen niveles bajos de testosterona (13). Ciertamente en este caso la coincidencia de comorbilidades, como obesidad, diabetes, síndrome metabólico y otros procesos, han oscurecido la evidencia (14). La enfermedad cardiovascular, fundamentalmente representada por la ateroesclerosis, es un largo proceso biológico en el que inicialmente puede haber signos exclusivos de disfunción, sin daño orgánico, y finalmente alteraciones estructurales reconocibles mediante técnicas de imagen. En los últimos año se ha demostrado que la disfunción eréctil reconocible en individuos sin evidencia de enfermedad cardiovascular orgánica, es un heraldo de la misma y este hallazgo ha renovado el interés por el potencial papel coincidente de la testosterona en la función endotelial y en las disfunciones endoteliales que fundamentan muchos de los trastornos de la erección (15).

La mortalidad femenina por enfermedad cardiovascular antes de los 50 años, es decir, antes de la menopausia, es la mitad que la del varón de la misma edad (16). Esto es debido al efecto beneficioso de los estrógenos. A partir de la menopausia los niveles plasmáticos de estrógenos son similares en el varón y en la mujer. Podría sospecharse también que la mayor mortalidad del varón es motivada por un efecto nocivo de los andrógenos. Efectivamente, dosis elevadas de andrógenos, en atletas y culturistas, aumentan el riesgo de infarto y accidentes vasculares cerebrales. Se producen también cambios en el perfil lipídico, progreso de la ateromatosis y disregulación 
de otros factores hemostáticos como es la reducción de la actividad fibrinolítica $(17,18)$. No está clara la relación entre testosterona e hipertensión; en experiencias animales la administración de andrógenos motiva hipertensión; entre la población añosa de Rancho Bernardo (19) los niveles bajos de testosterona (TDS) se asociaron con hipertensión y enfermedad coronaria.

\section{MECANISMO DE ACCIÓN DE LA TESTOSTERO- NA EN EL SISTEMA CARDIOVASCULAR}

El mecanismo de acción de la testosterona en su potencial efecto vaso dilatador coronario y periférico, ha sido investigando en experimentación animal y clínica humana mediante procedimientos diversos y aparentemente con conclusiones coincidentes: la testosterona potencia la vía del óxido nítrico en su acción sobre el endotelio. La información actual se ha conseguido, especialmente, investigando la relación existente entre testosterona, y masa muscular por un lado y testosterona y disfunción eréctil por otro.

\section{Músculo estriado y sarcopenia}

La testosterona ha sido utilizada abusivamente para mejorar el rendimiento físico de los atletas consiguiéndose la mejoría de la función muscular por su efecto anabolizante. Continúa siendo el recurso más común de aquellos individuos que desean mejorar su masa muscular, aunque sólo sea por simples razones cosméticas. El déficit de testosterona produce sarcopenia, un fenómeno especialmente interesante del varón que envejece ya que la debilidad muscular anuncia la fragilidad del anciano (FRAILTY), y es el inmediato predecesor de la discapacidad. Esta fragilidad está caracterizada por la pérdida de masa muscular y aumento de la grasa. Se ha demostrado que los andrógenos promueven la diferenciación de las células mesenquimales pluripotentes en células musculares, inhibiendo simultáneamente la diferenciación en la línea de células grasas. La terapéutica sustitutiva con testosterona invierte este proceso de pérdida de masa muscular e incremento de la grasa, típico del anciano. Esta respuesta se mantiene a pesar de la edad siendo el efecto anabólico de la testosterona similar en los individuos con edades comprendidas entre 60-75 años y los de 19-35 años. El grupo de trabajo de Bhasin et al $(20,21)$ ha demostrado que existen receptores androgénicos no sólo en las células musculares esqueléticas sino en otras células, como fibroblastos, células endoteliales, músculo liso, células plasmáticas y células satélites siendo éstas donde más receptores androgénicos se han encontrado. La administración de testosterona a varones añosos consigue hipertrofia del músculo esquelético, pero también se ha detectado incremento en las motoneuronas (22). En definitiva, es posible que el efecto de la testosterona no se limite sólo a la célula muscular esquelética sino que mejore su trofismo por estímulo de las motoneuronas, e incluso por un efecto indirecto, mejorando la vascularización del área músculo esquelética. Una acción sinergística, multifocal puede ser la responsable de la mejoría de la masa muscular.

\section{Músculo liso trabecular, endotelo y disfunción eréctil}

La interacción entre testosterona y endotelio peneano ha sido investigada en clínica y experimentación animal. Conocemos bien en la actualidad el mecanismo implicado en la erección, con el protagonismo del óxido nítrico via guanosinafosfato y guanilatociclasa. La investigación actual pretende conocer en qué puntos actúa la testosterona en este proceso, en el caso de que su actuación sea relevante. Existen varias opciones en el camino molecular de la función eréctil; potenciando algunos de los elementos moleculares que participan en esta función; sobre los receptores androgénicos, potenciando, inhibiendo o modulando los efectos inhibidores que participan en el proceso. Por otro lado la testosterona puede actuar directamente, a través de la DHT o por aromatización estrogénica.

La observación clínica ha podido precisar que no todos los niveles subnormales de testosterona plasmática actúan del mismo modo sobre los síntomas correspondientes a la disfunción sexual. En el estudio europeo sobre el varón añoso (EMAS) en el que se incluyeron 3369 varones con edades comprendidas entre 40-79 años, pudo comprobarse que los tres síntomas más relevantes, entre un grupo seleccionado de 31 síntomas potencialmente relacionados con la deficiencia androgénica, no todos se manifestaban al mismo tiempo, existiendo una cascada relacionada con los umbrales de testosterona plasmática. De este modo, cuando los niveles estaban en 12,10 $\mathrm{nmol} / \mathrm{I}$ el síntoma más llamativo correspondía a un desfallecimiento del vigor físico; cuando los niveles estaban en 10. $4 \mathrm{nmol} / \mathrm{l}$ desaparecían las erecciones matutinas; finalmente cuando los niveles estaban en $8.7 \mathrm{nmol} / \mathrm{l}$ desaparecía el deseo acompañado de disfunción eréctil (1).

\section{Experiencias animales}

La deprivación androgénica produce trastornos anatómicos y funcionales en diversas especies animales, efectos que han sido especialmente estudiados en ratas y conejos. En ratas produce reducción de fibras elásticas en la túnica albugínea y del músculo liso del cuerpo cavernoso (23). En el conejo, que después de la castración mantiene ciertos niveles de testosterona, se producen resultados similares, reversibles cuando tratados con testosterona (24). Por 
otro lado, la castración del conejo aumenta la cantidad de células grasas en la región subalbuginea reduciendo su elasticidad y pérdidas de músculo liso trabecular, con aumento del conectivo (25). Estas alteraciones tróficas y anatómicas de la pared vascular, del endotelio y músculo liso peneano, están motivadas por disregulación de la síntesis de VEGF,TGTbl y CTG (26). El efecto funcional de la testosterona sobre la dinámica eréctil se produce verosímilmente por acción directa ya que los inhibidores de la 5 alfa reductasa no producen impotencia estable. El mecanismo fundamentalmente implicado consiste en la mayor producción de la sintasa del óxido nítrico (NOSe) y también regulando la actividad y producción de la PDE-5 (27-30); no parece que exista un efecto neural directo.

En relación con el papel de los receptores androgénicos es conocido que la capacidad de fijación de la testosterona en estos receptores depende de su singular polimorfismo. Los receptores androgénicos tienen longitud de secuencias diferentes de GLUTAMINA y GLICINA y son precisamente aquellos que tiene menos repeticiones de GLUTAMINA los que fijan mejor los andrógenos. Esta afinidad de los receptores es más intensa para la DHT que para la testosterona. Con la edad los receptores androgénicos se alteran, motivo por el que se considera que las personas añosas seguramente necesiten un umbral de testosterona mayor para conseguir una función eréctil satisfactoria.

En definitiva, combinando hallazgos de experiencias animales y observación clínica puede concluirse el siguiente marco de interacción entre androgenos y función eréctil: regulan la producción y actividad de las isoformas de las sintasas del óxido nítrico (NOS); modulan la actividad de la PDE-5.

\section{ANDROGENODEFICIENCIA Y SALUD DEL VA- RON AÑOSO}

Hasta la pasada década la investigación clínica y analítica del estatus androgénico del varón en general, y más precisamente del varón añoso, en la consulta urológica, estaba presidida por sus potenciales interrelaciones con enfermedades prostáticas, seguimiento de los enfermos con cáncer de próstata tratados con deprivación androgénica, pacientes con disfunción eréctil, sin gran entusiasmo en este último caso, y algunas otras circunstancias minoritarias. Los progresos recientes han confirmado que el estatus androgénico del varón añoso, especialmente reconocido por la determinación de testosterona plasmática total, es un relevante marcador de salud general y, seguramente, de mayor valor mientras más elevada es la edad del individuo. Estos hallazgos han motivado dos efectos importantes desde el punto de vista sanitario y profesional; las determinaciones analíticas androgénicas, especialmente referidas a la testosterona total, aunque también a otros andrógenos, se solicitan ahora con muchísima más frecuencia que hace años (31); la detección de la androgenodeficiencia se reconoce con mucha más frecuencia y su interrelación con otros marcadores de salud general, y no sólo exclusivamente sexual, es ahora entendida y apreciada de una manera más relevante e integradora. En consecuencia la consulta urológica del varón añoso tiene ahora una perspectiva distinta, con un mayor contenido clínico y analítico y una visión más cabal e integradora de la salud del varón añoso. Si la mayor causa de muerte continúa siendo por motivos cardiovasculares, confirmado que la testosterona juega un papel importante en la salud vascular y endotelial, la investigación de la androgenodeficiencia en este grupo de población, se convertirá en un estudio inexcusable $y$, sin duda, justificará motivaciones para un renovado interés farmacológico de la testosterona, tanto con fines preventivos, en muy variados procesos, como terapéuticos y no solo por especialistas si no en Atención Primaria.

\section{CONCLUSIONES}

El estatus hormonal del varón a lo largo de toda su vida, fundamentalmente representado por los niveles plasmáticos de testosterona, mantiene muy variados compromisos fenotípicos, bien conocidos, emergiendo en la actualidad un papel en la estructura y función cardiovascular mucho más relevante que anteriormente sospechado. Niveles normales de andrógenos son necesarios tanto para la protección anatómica del sistema cardiovascular como para la función hemodinámica correcta. Niveles bajos de testosterona plasmática (TDS) han sido detectados en pacientes con mayor riesgo cardiovascular debido a ateroesclerosis aórtica o coronaria, síndrome metabólico, obesidad, hipertensión, diabetes tipo II y otras patologías sistémicas. La testosterona participa en la salud cardiovascular y hemodinámica, directa o indirectamente (DHT) por varios caminos: A nivel metabólico su deficiencia genera perfiles lipídicos proaterogénicos (aumento del nivel de colesterol y LDL, aumento niveles de triglicéridos, reducción niveles de $\mathrm{DHL}$ ) y disregulación de los mecanismos protectores fibrinolíticos facilitando, simultáneamente, la mayor actividad inflamatoria inherente a este proceso: A nivel anatómico protege la elasticidad vascular sistémica reduciendo el progreso de la ateroesclerosis, protegiendo el músculo liso vascular y el endotelio de tal modo que la castración produce apoptosis y rigidez de la pared arterial: A nivel funcional la tes- 
tosterona es vasodilatador sistémico y específico del terreno peneano.

Los mecanismos específicos por los que la testosterona contribuye a un buen control hemodinámico, cardiotónico, y vasodilatador en el territorio peneano, están en periodo de investigación pero ya se ha podido establecer que la testosterona, directa o indirectamente, participa en los mecanismos vasodilatadores endoteliales y del músculo liso trabecular facilitados por la via del óxido nítrico, promoviendo la producción de varias isoformas de la sintasa del óxido nítrico y fundamentalmente de la sintasa endotelial (NOSe), pero también potenciando la producción y acción de los inhibidores de la fosfodiesterasa 5 (PDE-5). El papel de los receptores androgénicos ha sido motivo de menor atención investigadora.

La testosterona, por lo tanto juega un papel en la salud del varón añoso mucho más importante de lo sospechado hasta ahora. Simultáneamente íntegra factores de riesgos en órganos y funciones (salud prostática, salud cardiovascular, salud sexual masa muscular, salud ósea, disfunción miccional, disfunción eréctil, sarcopenia, osteoporosis, etcétera) tradicionalmente considerados independientes. La determinación analítica de la testosterona se impone como inexcusable en la consulta urológica del varón añoso. Pero también de otros especialistas y muy verosimilmente de Atención Primaria.

\section{BIBLIOGRAFÍA y LECTURAS RECOMENDADAS (*lectura de interés $y^{* *}$ lectura fundamental)}

*1. Morales A, Heaton JPW, Carson CC. Andropause: A Misnomer for a true Clinical Entity. J. Urol. 2000; 163:706-712.

2. Vela Navarrete R. El Síndrome de Adam (Andropausia): Nueva perspectiva de un viejo síndrome clínico. Tiempos Médicos 570, 2000; 5-10.

*3. Schulman AUA Today, Morales A, Schulman C, Tustain J, Wu FCD. Testosterone deficiency syndrome (TDS) needs to be named appropriately: The importance of accurate terminology. EUR Urol 2006; 60:407-9.

4. Edwards E, Hamilton J and Duntley S. Testosterone propionate as a therapeutic agent in patients with organic disease of peripheral vessels, $\mathrm{N}$ Engl J Med 220 1939; p. 865-869.

*5. Hamm. Testosterone propionate in the treatment of angina pectoris, J Clin Endocrinol 2, 1942; p. 325-328.
*6. Levine AA and Likoff WB. The therapeutic value of testosterone propionate in angina pectoris, $\mathrm{N}$ Engl J Med 229 1943; p. 770-772.

7. Lesser MA. Testosterone propionate therapy in one hundred cases of angina pectoris, J Clin Endocrinol 6 1946; p. 549-557.

**8. English KM, St7.- eeds RP, Jones TH et al. Low dose tansdermal testosterone therapy improves angina threshold un men with chronic stable angina. Circulation 2000; 102: 1906-11.

9. Hak AE, Witteman JCM, De Jong FH et al. Low levels of endogenous increase the risk of atherosclerosis in elderly men: The Rotterdam Study, J. Clin Endocrinol Metab 2002; 87:3632-9.

*10. Muller M, Van Den Beld AW, Bots ML et al. Endogenous sex hormones and progression of carotid atheroseclerosis in elderly men. Circulation 2004; 109: 2074-9.

11. Larsen BA, Nordestgaard BG, Stender $\mathrm{S}$ et al. Effect of testosterone on atherogenesis in cholesterol-fed rabbits with similar plasma cholesterol levels. Atherosclerosis 1993; 99: 79-86.

*12. Araujo AB, Handelsman D. McKinlay JB. Total testosterone as a predictor of mortality in men: Results from the Massachusetts Male Aging Male Study. The Endocrine Society Annual Meeting. San Diego. CA USA Program and Abstract 2005; P1-561.

**13. Jones RD, Nettleship JE, Kappor D et al. Testosterone and atherosclerosis in aging men: purported association and clinical implications. Am J Cardiovasc Drugs 2005; 5: 141-54.

14. Jones TH. Testosterone Associations with erectile dysfunction, Diabetes, and the Metabolic Syndrome. European Urology Supp. 2007. 847-857.

**15. Montorsi P, Ravagnani PM, Galli S et al. Association between erectile dysfunction and coronary artery disease: matching the right target with the right patient. Eur. Urol 2006; 50: 721-31.

16. Barret-Connor EL. Testosterone and risk factors for cardiovascular disease in men. Diabetes Metabol 1995; 21:156-161.

17. Phillips GB, Pinkernell BH and Jing TY. The association of hypotestosternemia with coronary artery disease in men. Arterioscler. Thromb 1994; 14: 701-706.

18. Winkler UH. Androgens and hemostasis. Textbook of men's Health Parthenon Publishing 2002; 344-350.

19. Khaw K, Barret-Connor E. Blood pressure and endogenous testosterone in men: an inverse relationship. J. Hupertension 1988; 6:329-332.

20. Bhasin WE, Taylor and Singh $\mathrm{R}$ et al. The mechanisms of androgen effects on body composition: mesenchymal pluripotent cell as the target of androgen action, J Gerontol 58A 2003; p. 11031110. 
21. Bhasin S, Calof OM, Storer TW, Lee ML, Mazer NA, Jasuja R.et al. Drug insight: testosterone and selective androgen receptor modulators as anabolic therapies for chronic illness and aging. Nat Clin Pract 2006; 2: 146-59.

22. Keast JR, Gleeson RJ and Shulkes A et al. Maturational and maintenance effects of testosterone on terminal axon density and neuropeptide expression in the rat vas deferens, Neuroscience 112 2002; p. 391-398.

23. Shen $\mathrm{Z}$, Chen $\mathrm{Z}$ and Lu Y et al. Relationship between gene expression of nitric oxide synthase and androgens in rat corpus cavernosum, Chin Med J (Engl) 113 2000; p. 1092-1095.

*24. Traish A and Kim N. The physiological role of androgens in penile erection: regulation of corpus cavernosum structure and function, J Sex Med 2 2005; p. 759-770.

*25. Traish AM and Kim N. Weapons of penile smooth muscle destruction: androgen deficiency promotes accumulation of adipocytes in the corpus cavernosum, Aging Male 8 2005; p. 141-146.

26. Traish AM, Goldstein I, Kim NN. Testosterone and Erectile Function: From Basic Research to a
New Clinical Paradigm for Managing Men with Androgen insufficiency and Erectile Dysfunction. European Urology 2007; 52: 54-70

27. Lugg A, Rajfer A and Gonzalez-Cadavid NF. Dihydrotestosterone is the active androgen in the maintenance of nitric oxide-mediated penile erection in the rat, Endocrinology 136, 1995; p. 1495-1501.

28. Muller SC, Hsieh JT and Lue TF et al. Castration and erection. An animal study, Eur Urol 15, 1988; p. 118-124.

*29. Zhang X, Morelli H, and Luconi M et al. Testosterone regulates PDE5 expression and in vivo responsiveness to tadalafil in rat corpus cavernosum, Eur Urol 47, 2005; p. 409-416.

30. Morelli A, Filippi S and Mancina R et al. Androgens regulate phosphodiesterase type 5 expression and functional activity in corpora cavernosa, Endocrinology 146, 2004; p. 2253-2263

31. Martiíez Havaloyas JM, Quepo Zaragoza A, Ferrandis Cortes $\mathrm{C}$ et al. Cambios en las hormonas sexuales en varones mayores de 50 años. Prevalencia de niveles bajos de testosterona y factores de riesgo. Actas Urol. Esp. 2008; 32: 603-610. 